\title{
Metabolomics-Guided Insights on Bariatric Surgery Versus Behavioral Interventions for Weight Loss
}

\author{
Sara Tulipani ${ }^{1,2}$, Jules Griffin ${ }^{3,4}$, Magali Palau-Rodriguez ${ }^{1}$, Ximena Mora-Cubillos ${ }^{1}$, Rosa M. Bernal-Lopez ${ }^{5,6}$, \\ Francisco J. Tinahones ${ }^{2,6}$, Barbara E. Corkey ${ }^{7}$, and Cristina Andres-Lacueva ${ }^{1}$
}

Objective: To review the metabolomic studies carried out so far to identify metabolic markers associated with surgical and dietary treatments for weight loss in subjects with obesity.

Methods: The Preferred Reporting Items for Systematic Reviews and Meta-Analyses (PRISMA) guidelines were followed.

Results: Thirty-two studies successfully met the eligibility criteria. The metabolic adaptations shared by surgical and dietary interventions mirrored a state of starvation ketoacidosis (increase of circulating ketone bodies), an increase of acylcarnitines and fatty acid $\beta$-oxidation, a decrease of specific amino acids including branched-chain amino acids (BCAA) and (lyso)glycerophospholipids previously associated with obesity, and adipose tissue expansion. The metabolic footprint of bariatric procedures was specifically characterized by an increase of bile acid circulating pools and a decrease of ceramide levels, a greater perioperative decline in BCAA, and the rise of circulating serine and glycine, mirroring glycemic control and inflammation improvement. In one study, 3-hydroxybutyrate was particularly identified as an early metabolic marker of long-term prognosis after surgery and proposed to increase current prognostic modalities and contribute to personalized treatment.

Conclusions: Metabolomics helped in deciphering the metabolic response to weight loss treatments. Moving from association to causation is the next challenge to move to a further level of clinical application.

Obesity (2016) 24, 2451-2466. doi:10.1002/oby.21686

\section{Introduction}

Despite evidence to support their utility, lifestyle-based strategies for weight loss and treatment of obesity (i.e., based on diet and physical activity) have met so far with little success in the long term in terms of permanent weight loss (1). Bariatric surgery is the only current treatment for obesity leading to sustained weight loss (2) and to improvements in glucose regulation, up to a complete remission of type 2 diabetes (T2D) in the short and long follow-up (3-6). Consistent with the causative role of several organs being involved in metabolic homeostasis and both the development of T2D and obesity (e.g., including pancreatic islets, liver, fat cells, but also brain, gut, vasculature, muscle) (7-9), it is accepted that metabolic surgery (the remodeling of metabolism following weight loss) would act on a global rather than local scale through the restoration

\footnotetext{
${ }^{1}$ Department of Nutrition, Food Sciences and Gastronomy, Biomarkers \& Nutrimetabolomic Lab, XaRTA, INSA, Faculty of Pharmacy and Food Science, University of Barcelona, Barcelona, Spain. Correspondence: Sara Tulipani (sara.tulipani@ub.edu) and Cristina Andres-Lacueva (candres@ub.edu)

${ }^{2}$ Biomedical Research Institute (IBIMA), Service of Endocrinology and Nutrition, Malaga Hospital Complex (Virgen de la Victoria), University of Malaga, Malaga, Spain ${ }^{3}$ MRC Human Nutrition Research, Elsie Widdowson Laboratory, Cambridge, UK ${ }^{4}$ Department of Biochemistry and the Cambridge Systems Biology Centre, University of Cambridge, Cambridge, UK ${ }^{5}$ Biomedical Research Institute (IBIMA), Service of Internal Medicine, Malaga Hospital Complex (Hospital Regional Universitario de Malaga), University of Malaga, Malaga, Spain ${ }^{6}$ CIBER Fisiopatología de la Obesidad y Nutrición (CIBERobn), Instituto de Salud Carlos III (ISCIII), Madrid, Spain ${ }^{7}$ School of Medicine, Obesity Research Center, Boston University, Boston, Massachusetts, USA.
}

Funding agencies: This research was supported by: PI13/01172 Project (Plan N de I+D+i 2013-2016) co-funded by ISCIl-Subdirecciœn General de Evaluación y Fomento de la Investigación and Fondo Europeo de Desarrollo Regional (FEDER) PI-0557-2013 Project co-funded by Fundación Progreso y Salud, Consejería de Salud y Bienestar Social, Junta de Andalucía, and FEDER; JPI HDHL FOODBALL Project (PCIN-2014-133-MINECO-Spain); and 2014SGR1566 award from the Generalitat de Catalunya's Agency, AGAUR. S.T., X.M.-C., R.M.B.-L., and C.A.-L., respectively acknowledge the Juan de la Cierva fellowship (MINECO), the AGAUR for the predoctoral FI-DGR 2012 fellowship, the incorporation of doctors accredited research career in groups of Andalusian Public Health System (Consejería de Igualdad, Salud y Pollticas Sociales), and Ministry of Education, Culture and Sport for stays of mobility of professors in foreign centers of higher education and research.

Disclosure: The authors declared no conflict of interest.

Author contributions: S.T. and C.A.-L. designed the research; M.P.-R., X.M-C., and S.T. conducted the studies' eligibility assessment, the data extraction, and the table generation; S.T., M.P.-R., J.G., B.E.C., F.J.T., and C.A.-L. generated the discussion on the extracted data; S.T. wrote the paper; J.G., B.E.C., F.J.T., and C.A.-L. reviewed/edited the manuscript and proportionate a critical cut to the work though their scientific perspectives; S.T. and C.A.-L. are the guarantors of the study and have the primary responsibility for final content. All authors read and approved the final manuscript.

Additional Supporting Information may be found in the online version of this article.

Received: 11 June 2016; Accepted: 30 August 2016; Published online 28 November 2016. doi:10.1002/oby.21686 
of physiological pathways in a wide range of tissues and organs (10). However, a definitive explanation of its systemic effects is still lacking. Evidence also demonstrates that the remission of insulin resistance (IR) and T2D takes place even before significant weight loss has been achieved (within days to weeks after surgery) and does not necessarily associate either with the overall weight loss in the longer term or the surgical technique employed, confirming that other mechanisms beyond weight loss should explain both the rapid and sustained impact of the surgery on the metabolic improvements detected in individuals (11-13). Furthermore, the overall weight loss achieved by the different procedures did not differ in the longer term and was not significantly associated with T2D outcome; thus other mechanisms beyond weight loss should explain the rapid and sustained impact of malabsorptive techniques on IR and glycemic control (14).

Understanding the early and prolonged metabolic adaptations to both dietary and bariatric weight loss procedures may allow us to gain insights into shared and exclusive mechanisms of action of weight loss, help to dissect heterogeneity in response, identify individuals who would more likely benefit from moderate weight loss, predict prognosis, and ultimately guide personalized treatment (1521) (as reviewed in Ref. 22).

In this context, metabolomics - namely the comprehensive study of ideally all endogenous and exogenous metabolites contained in a biological system at a given moment-is currently considered as the most appropriate omics technology to investigate complex, polygenic, and multifactorial diseases with a strong multisystemic metabolic nature (23) such as obesity and T2D $(22,24-27)$.

The primary objective of this article was to review the metabolomic studies carried out so far to identify metabolic markers of response to surgical and dietary treatments for weight loss, according to the Preferred Reporting Items for Systematic Reviews and MetaAnalyses (PRISMA) guidelines.

\section{Methods}

\section{Data sources}

Two electronic databases (PubMed and Web of Science) were searched for keywords (details in Online Supporting Information). Species (human), language (English), and publication date restrictions (from 2000 to date, last search on 4th December 2015) were imposed. Relevant references identified from pertinent articles were additionally reviewed.

\section{Study selection}

Types of interventions, study designs, and participants. Prospective intervention trials carried out to study the metabolic response to surgical and/or dietary treatments for weight loss in adult subjects with overweight or obesity (males and females, BMI $>25 \mathrm{~kg} / \mathrm{m}^{2}$ ) were included. Randomization was expected only for parallel-arm lifestyle intervention trials (surgical procedures being applied according to clinical decision).

Analytical approach. Targeted and untargeted metabolomic approaches driven by mass spectrometry (MS) and proton nuclear magnetic resonance spectroscopy $\left({ }^{1} \mathrm{H}-\mathrm{NMR}\right)$ techniques were both included in the selection.
Types of outcome measures. Low-molecular-weight $(<1,000$ Da) metabolites significantly up- or downregulated following surgical or behavioral weight loss interventions, with respect to baseline and to the eventual parallel intervention, were the primary outcome measures of interest of the review. In the case of single-arm (quasi) trials, the post-intervention changes with respect to baseline were of primary interest, while baseline differences with respect to controls (i.e., lean healthy subjects) were not considered since they were not universally available. Weight loss was the cutoff clinical outcome required for inclusion in the review, except for acute postsurgery studies. In the case of parallel-arm studies, only those metabolites that differed systematically post-intervention (within-group variation during follow-up with respect to baseline) and between interventions (between-group variation) were considered as potential biomarkers of a given intervention, to minimize potential confounders. In the case of multi-time point followup studies, only metabolites that significantly differed from baseline at any follow-up point were considered in the summary tables. Additional clinical outcome measures such as known descriptors of glycemic control [i.e., fasting glucose, glucose tolerance, IR-HOMA (homeostasis model assessment), HbAc1b] were reported, when available.

\section{Data extraction}

Eligibility assessment was carried out independently by two authors using predefined data fields in an unblinded standardized manner. A third author conducted an independent review of the extracted articles, and cases of disagreements were resolved by majority consensus.

\section{Results}

Of the 304 studies initially retrieved, 32 successfully met the eligibility criteria for inclusion in the review (Figure 1).

\section{Study designs}

As summarized in Tables 1 and 2, studies ranged from parallel-arm trials comparing over time the effects of surgical versus dietary interventions or of different treatments of the same category (surgical only and behavioral only) up to the analysis of single-arm antiobesity interventions in respect to baseline. Only 2 of the 32 selected studies included an independent cohort in the study design to replicate/validate the obtained findings $(28,29)$.

\section{Participants}

The majority of the studies consisted of small-scale trials $(n=6-71$ participants). Only one large-scale metabolomic study $(n=500)$ has been carried out so far to investigate the effect of a combined behavioral intervention (diet and exercise) on subjects with obesity (validation cohort of the WLM trial) (29). The participation of women was generally higher, or even exclusive (29-33), but genderdependent variation in the metabolic response to treatment was only discussed in three studies $(29,34,35)$.

\section{Interventions}

Gastric bypass (Roux-en-Y, RYGB) was the most widely investigated among surgery procedures $(28,29,33,36-53)$, followed by sleeve gastrectomy $(30,40,48,53,54)$, adjustable gastric banding $(36,39)$, and biliopancreatic diversion with duodenal switch (BDDS) $(41,49)$. Behavioral weight loss strategies mainly consisted of 


\section{PRISMA 2009 Flow Diagram}

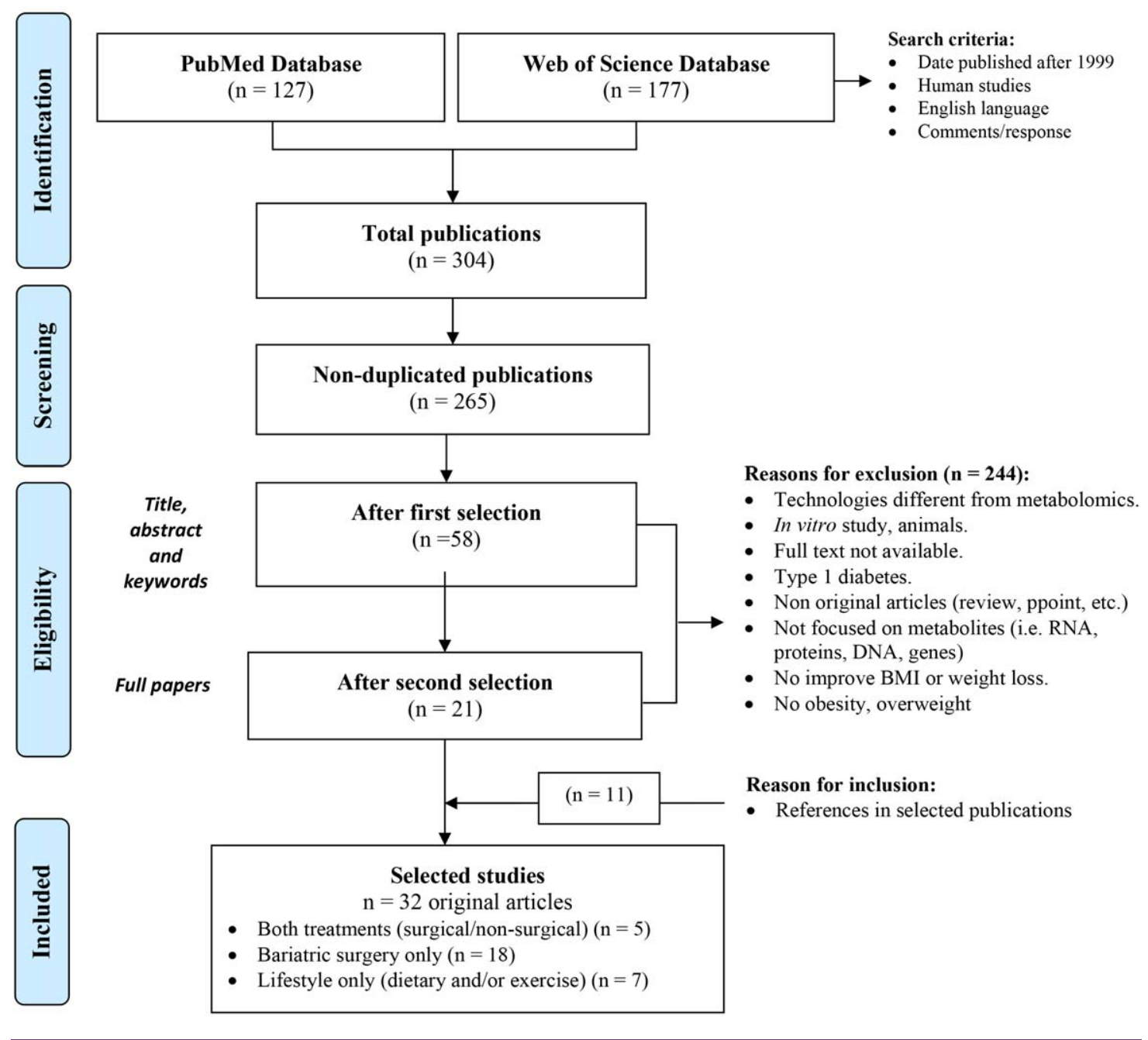

Figure 1 Schematic overview of the search strategy for this review. [Color figure can be viewed at wileyonlinelibrary.com.]

randomized controlled dietary intervention trials, and only three studies also included progressively increasing physical activity in a lifestyle treatment plan $(29,32,55)$. Except for sporadic exceptions (56), hypocaloric diets were the most common interventions, albeit with a variable degree of caloric restriction (i.e., -600 to $-1,200$ $\mathrm{kcal} /$ day) $(30,31,35,54,55)$, personalization (i.e., $-15 \%$ of daily energy requirements) (57), and modification of the macronutrient proportion (i.e., low- and very-low-carbohydrate diets, high vs. low protein intake). In a few cases, weight loss was achieved by adopting a meal replacement diet and through the distribution of specific dietary items to all subjects during weekly visits $(28,58)$.

\section{Follow-up and bio-specimens}

The duration of the interventions, as well as the types of specimens and the collection frequency varied among studies, being a relevant source of heterogeneity. Three bariatric studies investigated acute postsurgical effects ( $\leq 7$ days postoperatively) $(40,42,48)$ as these studies focused on the cause of T2D remission rather than the effect. The majority of the works focused on mid-term ( $\leq 6$ months) metabolic adaptations, while long-term effects $(\sim 1$ year $)$ have rarely been described to date $(n=4)(41,47,52,53)$.

Fasting blood serum and plasma collected in a fasted state were the most frequently accessed biological matrices, followed by urine (spot or 24 hr). Finally, the metabolomic profiling of dysfunctional tissues has been rare to date (55), probably due to invasiveness issues of taking biopsies.

\section{Clinical outcomes}

The outcomes of parallel-arm and single-arm designed studies are separately summarized in Supporting Information Tables S1 and S2. 


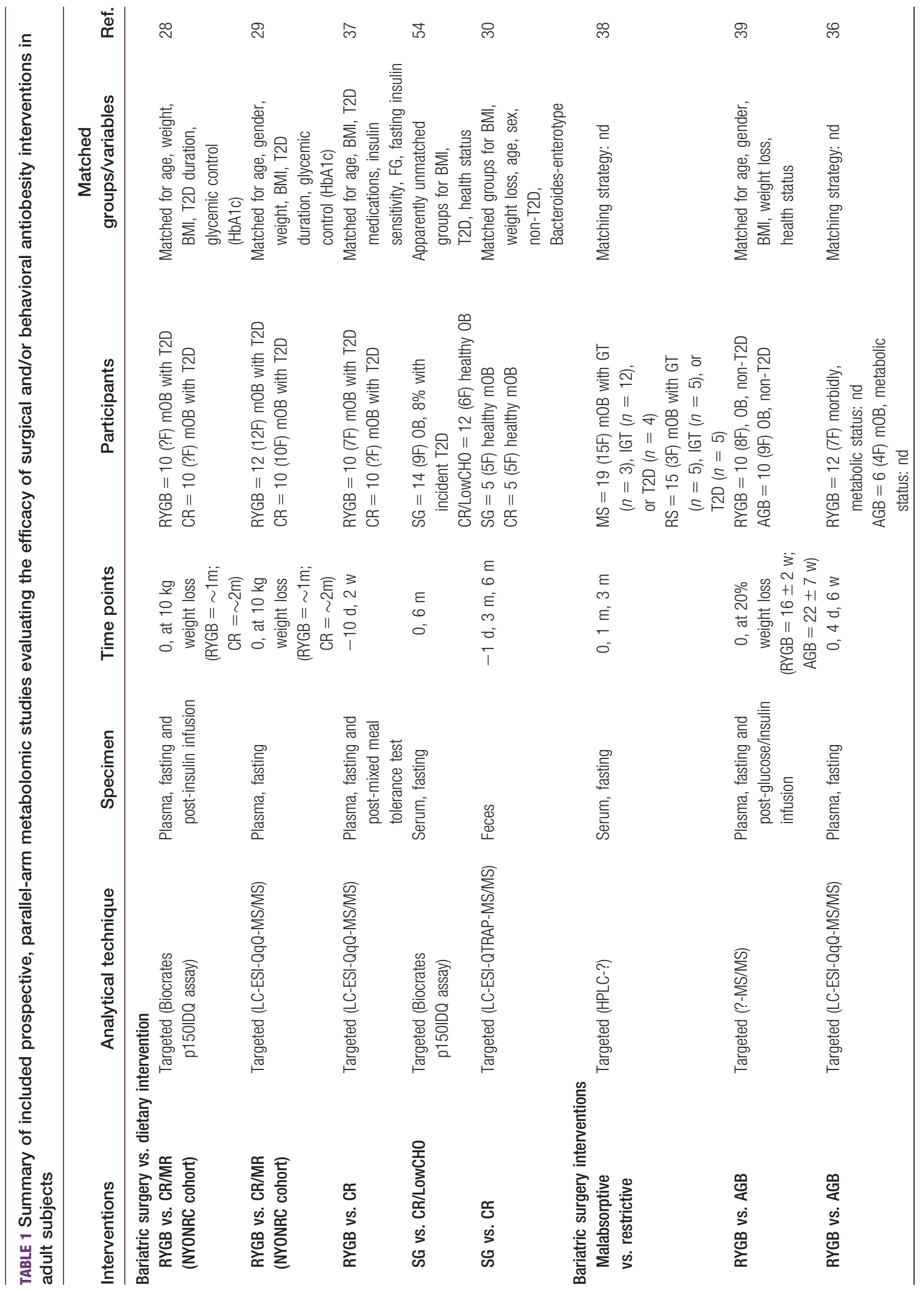




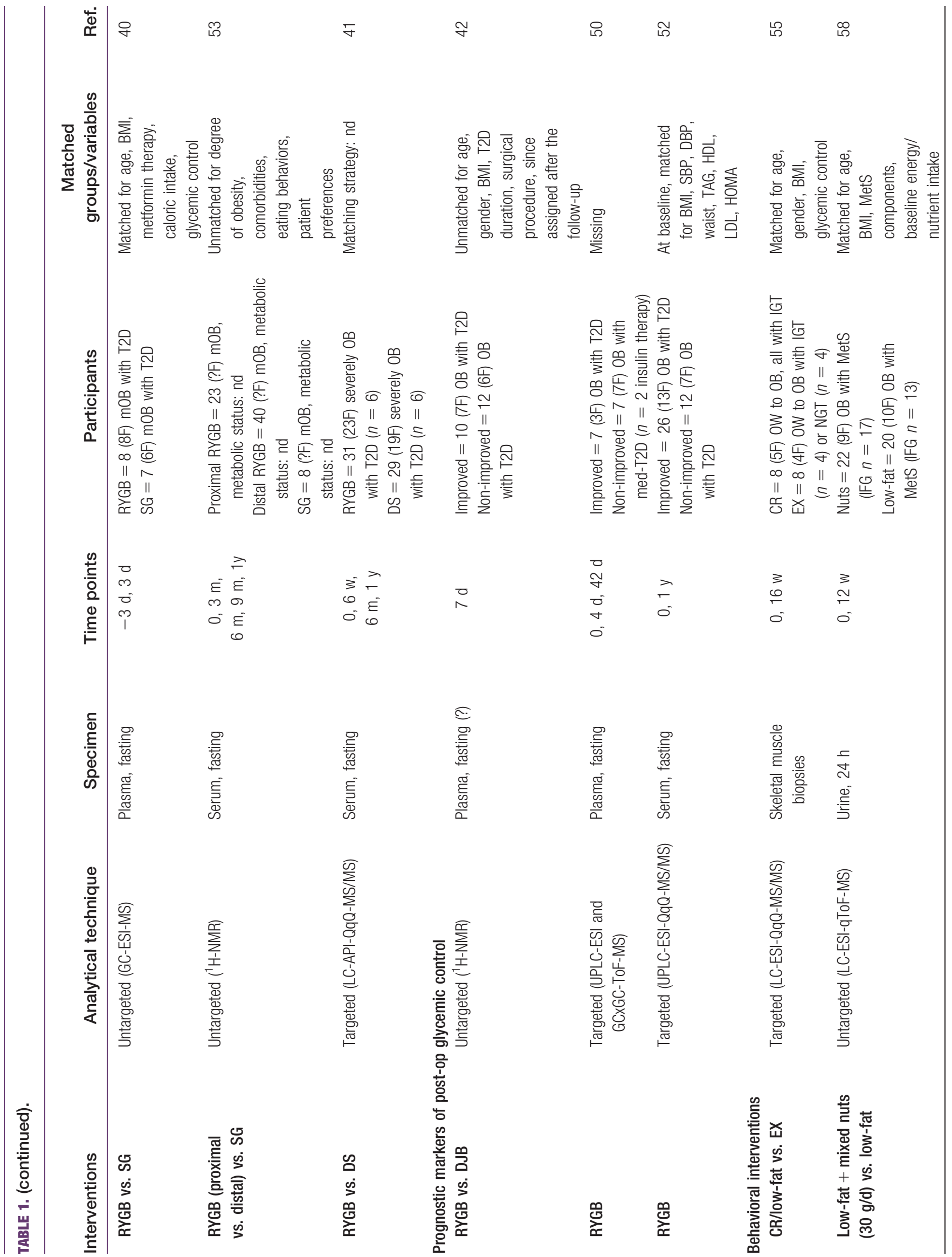




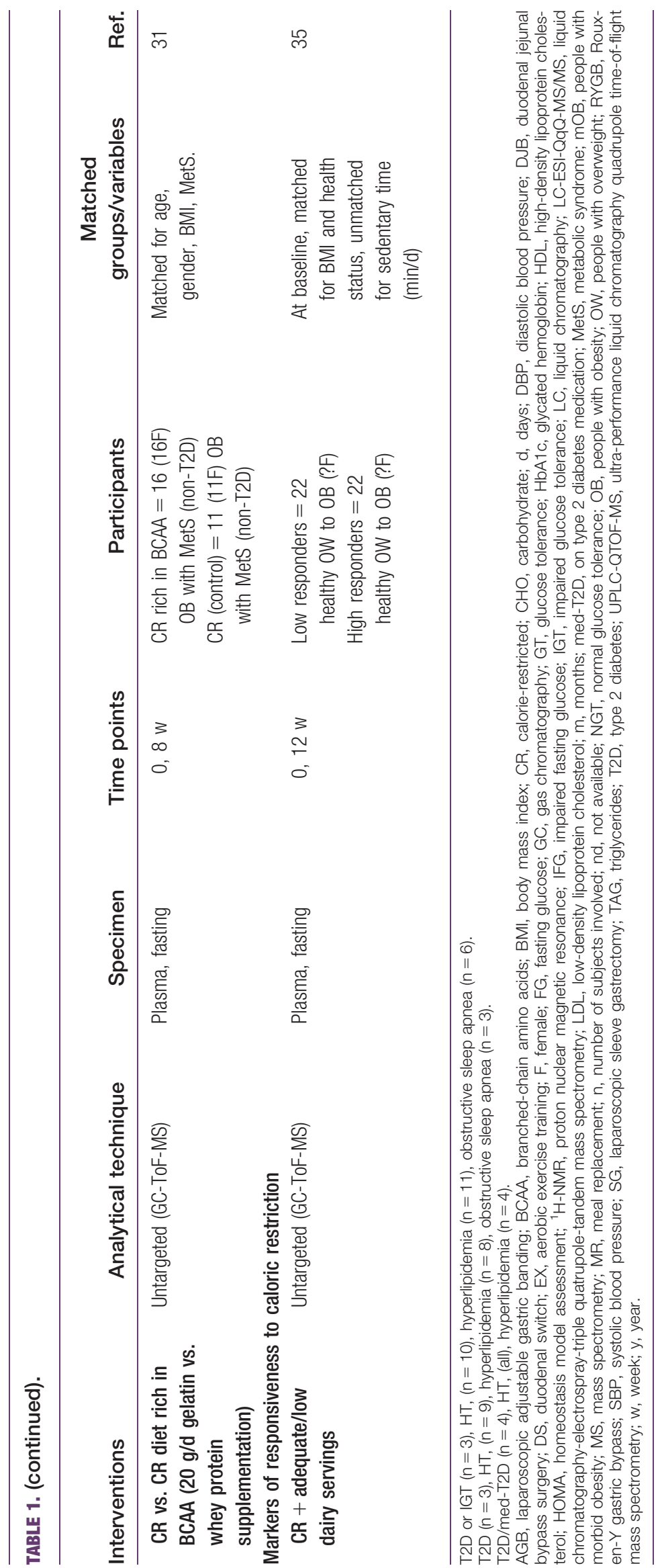




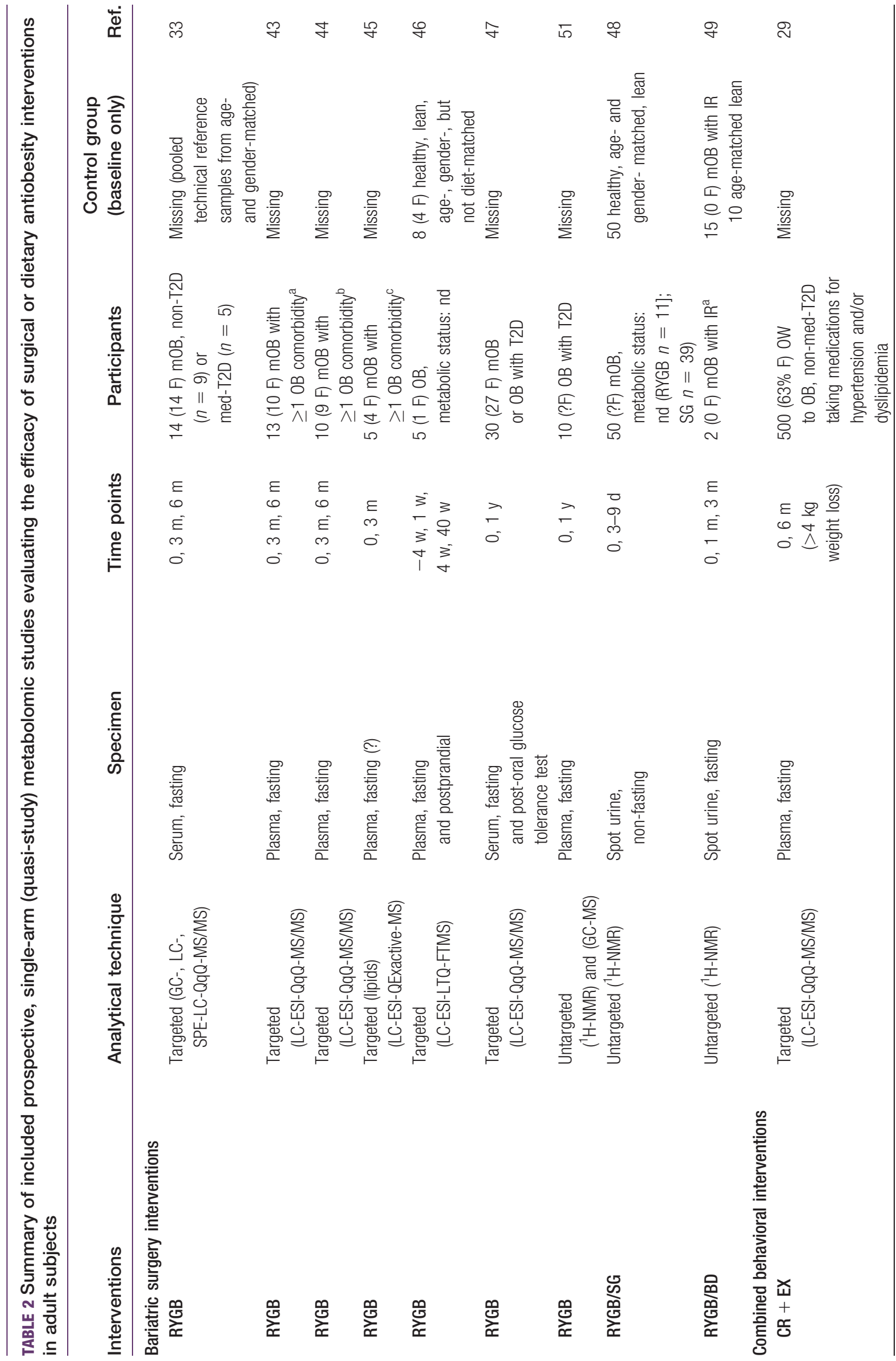




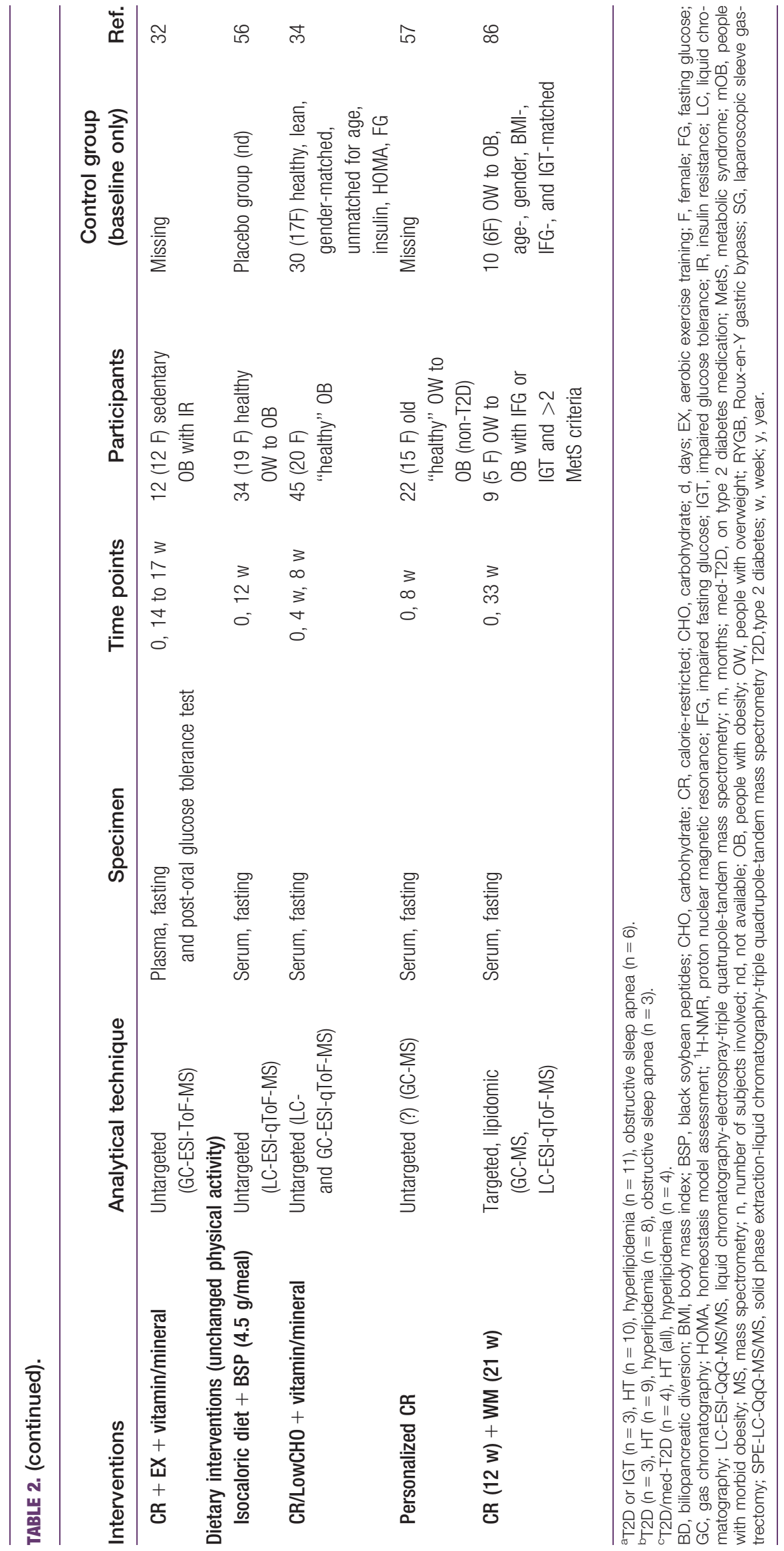


Besides BMI, measures of glycemic control were the most accessible criteria for defining the metabolic phenotypes in a study at baseline (Tables 1 and 2). Significant improvements in glycemic control (i.e., reduction of circulating $\mathrm{HbA} 1 \mathrm{c}$ concentrations, fasting/postprandial insulin, fasting/postprandial HOMA) and a more favorable plasma lipid profile (i.e., increased high-density lipoprotein cholesterol) were commonly observed, confirming the metabolic benefits of weight loss.

\section{Metabolomic approaches}

Mass spectrometry combined with different metabolite chromatographic separation (i.e., ultra-performance liquid chromatography, high-performance liquid chromatography, and gas chromatography) was the most commonly used analytical technique, followed by ${ }^{1} \mathrm{H}$ NMR-based approaches or by the integration of multi-platform analyses (54). An array of targeted and untargeted metabolomic approaches was applied to date, but targeted analysis predominated.

The two distinct approaches are complementary and, if both are not used, the choice between them should depend on the aim of each study. Untargeted metabolomics is the most comprehensive analysis of all the measurable analytes in a sample, including chemical unknowns. Consequently, among its intrinsic limitations, it provides wider opportunities for novel metabolite species, pathway, and target discovery (59). In turn, targeted metabolomics is reliant on a priori selection of a subset of biochemically characterized and interpreted metabolites, which often belong to selective established pathways (reduced coverage). Although the targeted approach allows quantitative results, reduces the likelihood of analytical artifacts, and eases up the troubles related to big data analysis and interpretation, the clear definition of the species measured hinders the discovery of novel metabolic perturbations (hypothesis-driven approach).

\section{Discussion}

\section{Bariatric versus dietary approaches: shared metabolic adaptations}

To our knowledge, only five parallel-arm metabolomic studies have directly compared so far the body systemic response with dietary and surgical treatments for weight loss (28-30,37,54), and groups were well matched only in two of them (for age, body weight, BMI, diabetes duration and management, and also extent of body weight lost during treatment, in $\mathrm{kg}$ or $\%$ loss $)(28,29)$. From a clinical viewpoint, in all cases the rate of weight loss and reduction of fat mass were faster after the surgery than the dietary changes, together with a more robust impact on glucose homeostasis improvement (28). However, the amount of weight loss was neither solely nor primarily correlated with improvement in IR. As summarized in Table 3, the metabolic adaptations shared by surgical and dietary interventions involved a decrease of several amino acids including branched-chain amino acids (BCAAs), together with an increase of total circulating ketone bodies and acylcarnitines. In contrast, bariatric surgery was associated with a greater decline in circulating BCAAs leucine, isoleucine, and valine than after an equivalent weight loss induced by diet (28), and postsurgical changes were reported soon perioperatively. The decrease of BCAAs and their related metabolites correlated with weight loss after RYGB only.
When including results from single-arm studies in the comparison, the depicted differential metabolic adaptations were confirmed (Supporting Information Table S3). Considering lipid metabolites, both surgical and dietary approaches promoted an overall decline of circulating (lyso)phospholipid species. Despite large-scale metabolomic studies such as those focused on the EPIC cohort recently indicating several choline-containing phospholipids as potential biomarkers of T2D $(26,27)$, the heterogeneous response of this wide lipid class to both treatments hampered the generation of mechanistic explanations for phospholipids. Bariatric procedures were most associated with a significant decline of circulating ceramides $(33,43,44)$, while dietary weight loss programs mainly reported the decrease of long- and very-longchain fatty acids with variable degrees of saturation, both in the free and esterified form (diacylglycerols and triglycerides) $(34,45,55,57)$.

\section{Restoration of protein metabolism}

$B C A A$. Following both types of weight loss programs, the circulating BCAAs were described as decreasing significantly, associated with improvement in IR and, more importantly, predicted metabolic benefits independently from the amount of weight lost. Although obesity-associated hyperaminoacidemia and the rise in circulating BCAAs are the most widely described shift in protein metabolism associated with obesity and IR, in both adults and childhood (6062 ), the mechanism(s) which may underlie an imbalance of protein turnover are numerous and remain unclear.

Overall, the decrease in circulating plasma BCAAs observed following the different weight loss strategies may result from (i) a decrease in protein intake (increased AA catabolism), (ii) a decrease in protein catabolism, observed to be secondary to increased insulin sensitivity, indicative of metabolic amelioration, (iii) an attenuation of tissue-specific alterations in the BCAA metabolism and a promoted amino acid uptake and tissue utilization (increased tissue BCAA catabolism), or (iv) a combination of these factors. Although BCAAs are found in dietary proteins and, therefore, diet remains a potential contributor to the modulation of their peripheral levels, previous studies have suggested that dietary intake accounts for only a small portion of blood BCAA and for their change during weight loss $(29,63)$. Furthermore, the more drastic decrease in circulating AAs detected following surgery should not depend on a decrease in protein intake as both surgical and dietary treatments actually shared a certain degree of caloric restriction, and the decrease in non-proteic amino acids such as ornithine and cystathionine would confirm these suppositions (Supporting Information Table S3). Consequently, other factors clearly influence the observed variations in BCAAs.

Reversion of tissue-specific alterations in BCAA metabolism could contribute, at least in part, to the decline of plasma BCAAs following weight loss treatments. This hypothesis makes sense since the surgery was accompanied by a consistent significant decline of specific products of incomplete mitochondrial oxidation of BCAAs, namely short-chain acylcarnitines C3 and C5 $(28,39,64)$. Furthermore, the activity of key BCAA catabolic enzymes is known to be altered in obesity, in a tissue-specific fashion with alterations in liver and adipose tissue but not in muscle, and these enzymatic changes contribute to the rise in plasma BCAAs (65). In turn, a strong correlation between the expression of BCAA catabolic genes, IR, and T2D has also been demonstrated in humans, and evidence exists of the reversion of the enzymatic alterations in adipose tissue 
TABLE 3 Shared and unique metabolic variations observed in parallel-arm bariatric surgery versus dietary intervention studies $^{a}$

\begin{tabular}{|c|c|c|c|c|}
\hline Metabolite & \multicolumn{2}{|c|}{ Variation $^{\mathrm{b}}$} & Class/function summary & Ref. \\
\hline 3-hydroxybutyrate & $\uparrow \uparrow$ & $\uparrow \uparrow$ & Ketone body & 28,37 \\
\hline Total ketones & $\uparrow \uparrow$ & $\uparrow \uparrow$ & Ketone bodies, molar sum & 28,37 \\
\hline Phenylalanine & $\downarrow \downarrow \downarrow$ & $\downarrow$ & Glucogenic and ketogenic AAA & $28,29,37$ \\
\hline Leucine & $\downarrow \downarrow$ & $\downarrow$ & Exclusively ketogenic BCAA & 28,29 \\
\hline Valine & $\downarrow \downarrow \downarrow$ & - & Glucogenic BCAA & 28,37 \\
\hline Alanine & $\downarrow \downarrow \downarrow$ & $\downarrow \downarrow$ & Glucogenic non-essential AA & $28,29,37$ \\
\hline Asparagine/aspartate & - & $\downarrow$ & AA ratio & 28 \\
\hline Glutamate & $\uparrow$ & - & Glucogenic AA & 54 \\
\hline Ornithine & $\downarrow \downarrow$ & $\downarrow$ & Others, non-proteic AA & 28,29 \\
\hline totBCAAs & $\downarrow \downarrow$ & - & Molar sum & 28,37 \\
\hline totAAAs & $\downarrow$ & - & Molar sum & 37 \\
\hline totAAs & $\downarrow \downarrow$ & - & Molar sum (proteic AAs) & 28,37 \\
\hline $\mathrm{C} 2$ & $\uparrow \uparrow$ & $\uparrow \uparrow$ & Short-chain ACs & 28,37 \\
\hline $\mathrm{C} 3+\mathrm{C} 5$ & $\downarrow \downarrow$ & $-\downarrow$ & Short-chain ACs (BCAAs catabolism) & 28,37 \\
\hline totACs & $\uparrow \uparrow$ & $\uparrow \uparrow$ & Molar sum & 28,37 \\
\hline $\mathrm{C} 4-\mathrm{OH}$ & $\uparrow$ & $\uparrow$ & Short-chain ACs & 28 \\
\hline Ci4-D/C4-DC & $\downarrow$ & - & Short-chain ACs (BCAAs catabolism) & 28 \\
\hline C16, C18:1 & $\uparrow$ & $\uparrow$ & Long-chain (even-) ACs & 28 \\
\hline C18:2 & $\uparrow \downarrow$ & $\uparrow-$ & Long-chain (even-) ACs & 28,54 \\
\hline PC aa C42:0 & $\downarrow$ & - & Glycerophospholipids with long- & 54 \\
\hline PC aa $32: 0, P C$ aa $32: 1, P C$ aa $40: 5$ & - & $\downarrow$ & Glycerophospholipids with long- & 54 \\
\hline
\end{tabular}

${ }^{a}$ All changes have been investigated in plasma/serum samples, collected in the fasted state.

$\mathrm{b}_{\uparrow}$ and $\downarrow$ respectively indicate a significant post-intervention increase or decrease in relative metabolite concentration, in respect to basal levels.

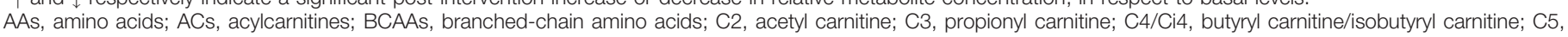

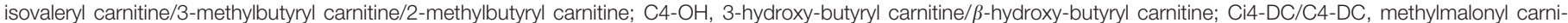

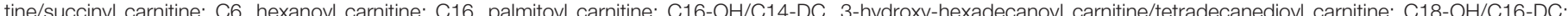

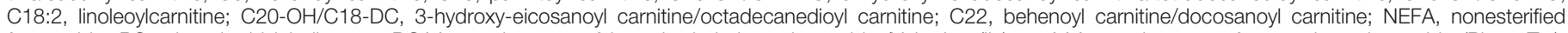

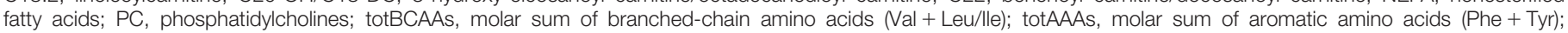
totAAs, molar sum of all amino acids measured.

BCAA metabolism by bariatric surgery-induced weight loss in subjects with obesity (66).

Other amino acids. Metabolic surgery was associated with a more drastic decrease in the total amino acid pools than that detected in dietary weight loss programs, particularly driven by the significant decline of the aromatic amino acids phenylalanine and tyrosine (AAAs), as well as ornithine, proline, and histidine (Table
3) $(28,37)$. Subjects with obesity had approximately twofold higher serum phenylalanine concentration than control subjects, which has been proposed as a noninvasive marker of liver dysfunction in obesity, related to liver steatosis. Consequently, AAAs have been proposed as additional markers of metabolic improvement directly or indirectly involved in postoperative adaptations (67).

Surprisingly, the decline of circulating alanine was even more frequently described than the decrease in BCAAs, especially as a 
response to surgical procedures. Although the mechanistic explanation is still unknown, plasma alanine levels may decrease in association with reduced dietary intake (i.e., through a low-protein diet) or vitamin deficiency (i.e., alanine metabolism is highly dependent upon enzymes having vitamin B6 as a cofactor) or even a decrease in fasting glucose available (68). Alanine is in fact an important substrate and regulator for glucose metabolism, playing a key role in glucose-alanine cycle between tissues and liver. In muscle and other tissues that degrade amino acids for fuel, alanine is produced during BCAA metabolism and most commonly via reductive amination of pyruvate, a product of muscle glycolysis. The alanine formed then is passed into the blood and transported to the liver, where a reverse of the alanine aminotransferase reaction takes place, and pyruvate regenerated forms glucose through gluconeogenesis, which then returns to muscle. According to this latter scenario, decreased circulating levels of alanine could also indirectly reflect a depletion of pyruvate available in muscle for conversion (increased local oxidation) in respect to what would be expected in the "well fed" state, and this has been associated with blood sugar levels in both diabetes and hypoglycemia.

Finally, despite the overall decreasing aminoacidemia normally associated with obesity, circulating levels of other amino acids such as glycine have been shown to significantly increase after weight loss treatments, and especially following bariatric techniques (Supporting Information Table S3). Low fasting levels of glycine have been observed in individuals with impaired glucose tolerance and proposed as an early marker for IR (27). The rise in glycine may be associated with the positive metabolic effects of weight loss treatments, since it is involved in many different biochemical mechanisms including the regulation of plasma cholesterol and triglyceride levels as well as the restoration of antioxidant glutathione synthesis and reduction of tissue and oxidant damage (69). However, results are still contradictory, as are any eventual causative associations.

\section{Exclusive metabolic shifts after surgery}

Five parallel-arm metabolomic studies have focused so far on differentiating the metabolic response to malabsorptive [RYGB and duodenal jejunal bypass surgery] versus restrictive surgeries (laparoscopic sleeve gastrectomy and laparoscopic adjustable gastric banding), and only two of them clearly defined the group-matching strategy $(38,39)$. As shown in Table 4 , the main bariatric-specific metabolic response included a significant increase in circulating bile acids (BAs). A decrease in BCAAs valine, leucine, and isoleucine and other glycogenic amino acids (i.e., proline, histidine), the increase of serine and glycine, and a heterogeneous variation in the circulating acylcarnitines were also observed. The very similar metabolic signatures obtained in response to diversionary and nondiversionary surgeries suggest that the metabolic effects are downstream of the primary effects of the surgery despite the different approaches. This was consistent with clinical outcomes. Although a trend of greater improvement in glycemic control was associated with malabsorptive procedures (i.e., significant reduction of fasting glucose and fasting insulin and increase in plasma FGF19 levels), inter-study variation was apparent and hence hampered the definition of clear-cut differences among bariatric techniques.

\section{Restoration of BA metabolism}

Altered BA metabolism has been long observed in obesity and $\mathrm{T} 2 \mathrm{D}$, with a significant decline in the circulating BA pool, especially postprandially (70). In contrast, an increase in circulating BAs in humans has been observed following surgical treatment for obesity, including perioperative $(<7$ days $)$ and both in the fasted $(13,38,47)$ and postprandial state (46). Accordingly, BA secretion has been associated with improvement in insulin secretion, insulin sensitivity, and whole-body glucose homeostasis, as well as improving liver and pancreatic function in animal models of obesity (reviewed in Ref. 71). Malabsorptive techniques were expected to have a greater impact on BA metabolism, due to the anatomical adaptive changes associated with the surgery, including bile delivery to the terminal ileum, decreased enterohepatic BA circulation followed by increased conversion of cholesterol to BAs (13). Nevertheless, the currently available metabolomic studies give insights for a restoration of BA synthesis also sustained by restrictive techniques $(30,38)$. Nevertheless, the high variability in the study designs and the variable change in the BA pool and composition over time made it difficult to pick the nuances of BA changes after dinstict bariatric procedures.

Far from simply being dietary fat emulsifiers and the primary route governing cholesterol homeostasis, BAs have been recognized over the past decade as nutrient-responsive hormones that modulate various metabolic pathways through cell surface and nuclear receptors, including their own synthesis and enterohepatic circulation, but also triglyceride, glucose, and energy homeostasis (reviewed in Ref. 72). According to the most recent hypothesis, changes in bile flow after the surgery may have a direct role in IR amelioration via (i) increased satiety gut hormone responses (i.e., enhancing glucagon like peptide-1 response by L-cell stimulation), leading to reduced food intake and weight loss, (ii) inhibition of gluconeogenesis in a farnesoid $\mathrm{X}$ receptor-dependent and -independent manner, or (iii) insulin signaling promotion and glycogen synthase activation, thus aiding insulin-dependent control of glucose metabolism in the liver.

Moreover, preliminary evidence has shown that not all BAs act equally. For instance, Simonen et al. (47) postulated that altered conjugation of BAs after surgery is the actual mediator of metabolic consequences, independent of changes in total serum BAs, suggesting the role of gut microbiota, a key regulator of BA conjugation and secondary BA formation, in the metabolic adaptations observed postsurgery (73). In line with this hypothesis, a significant increase in the bile salt glycine/taurine conjugation ratio was also observed postoperatively (46).

\section{Early prognostic markers of metabolic flexibility: Ketone bodies}

Compared with behavioral antiobesity strategies, bariatric surgery is known to trigger very early adaptations. Hence, it is theoretically possible to identify early prognostic markers of the long-term metabolic response, particularly desirable to help clinicians in deciding whether drug therapy is necessary shortly after surgery and ultimately contributing to personalized treatment for obesity.

A NMR-based metabolomic analysis was specifically applied to differentiate the response to metabolic surgery according to diabetic improvement (42). The analysis showed that the metabolite profile of two groups ("improved" vs. "non-improved" diabetic individuals with obesity) differentiated at an early postoperative stage (7 days) leading to an accurate prognosis prediction of long-term glycemic 
TABLE 4 Shared and unique metabolic variations observed in parallel-arm malabsorptive versus restrictive surgery studies ${ }^{a}$

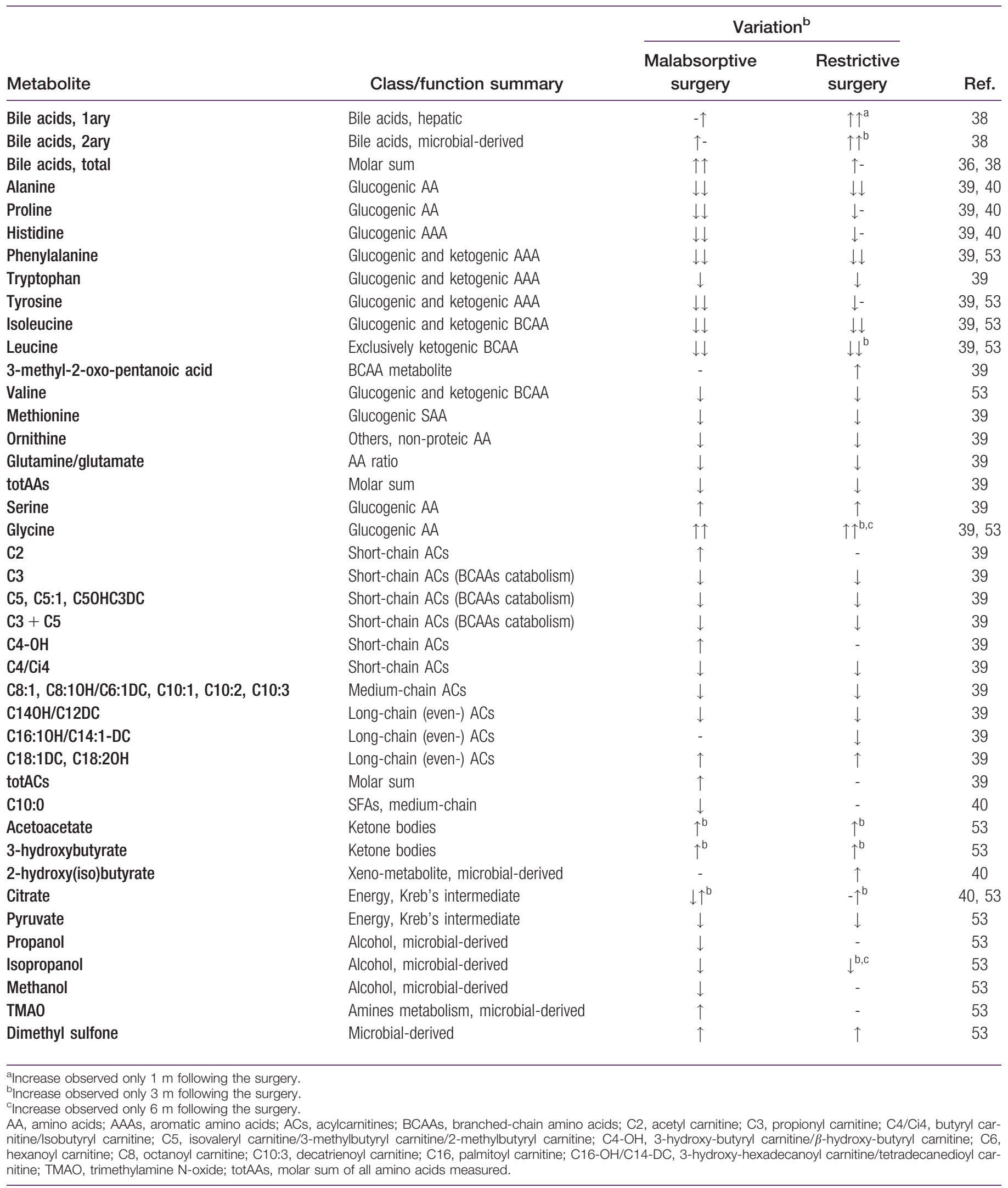


control after surgery (defined through HbA1c values at 3 months). Circulating 3-hydroxybutyrate (3-HB), higher in the improved group, was the most relevant early metabolic feature to positively correlate with a better glycemic control subsequently, together with lower glucose and lipid (low-density lipoprotein, very-low-density lipoprotein) concentrations. The $[3-\mathrm{HB}] /[$ glucose] ratio was observed to augment current prognostic modalities and proposed to help clinicians in deciding whether drug therapy is necessary shortly after surgery, ultimately contributing to personalized treatment.

Although starvation-associated ketoacidosis has been proposed as an explanation for the observed changes (increased fatty acid oxidation during perioperative fasting or caloric restriction) (74), this does not explain the selective increase in 3-HB in subjects with a better glycemic prognosis in the long term, since both the improved and nonimproved groups underwent the surgical stress or fasting immediately after surgery. A direct effect of 3-HB and ketone bodies on insulin sensitivity has been already shown (75); thus its rise in the early days after bariatric surgery might directly mediate glycemic control enhancement. As 3-HB is formed primarily when the energy source for the peripheral organs is shifted to lipid from glucose often during longer-term fasting, these alterations in metabolism at an early-stage postoperation may be a key factor in the success of longer-term postoperative glucose control. Increases in 3-HB may also reflect a change in the mitochondrial redox state of the liver (from a more oxidative state toward a more reduced state), which would be induced by the surgery only in patients with better responses in terms of their metabolic phenotypes (metabolic flexibility). The rise of circulating metabolites known to alter the liver redox state, including branched-chain ketoacids, BCAAs, and free fatty acids, recently associated with obesity and T2D (75), would support this last hypothesis, in keeping with the recent concept of redox as a master regulator of metabolism (76-78).

\section{Restoration of lipid metabolism}

From traditional clinical lipid measures, it is widely appreciated that dyslipidemia and abnormal lipid metabolism are characteristics of obesity, especially in association with abnormal glucose metabolism, i.e., impaired fasting glucose, impaired glucose tolerance, and T2D, while weight loss improves the blood lipid profile. Although lipidomics is now considered a self-standing "omics" technology, studies focused on the comprehensive analysis of lipid diversity were included in this review, due to the overlapping between the metabolomic and lipidomic approach and the strong impact of obesity and weight loss on all traditional lipid homeostasis measures $(79,80)$.

Decline of proinflammatory ceramides. A significant decrease in plasma ceramides was observed in diabetic subjects with obesity after RYGB, in conjunction with significant improvements in cardiovascular risk factors and insulin sensitivity (43). This study was the first to provide in vivo evidence following surgically induced weight loss. Subsequently, Graessler et al. (45) found a significant positive correlation between the RYGB-induced decrease of ceramide metabolites and the postoperative levels of $\mathrm{HbA} 1 \mathrm{c}$, together with triglycerides, total cholesterol, and low-density lipoprotein cholesterol. An increasing number of cell systems and animal and human studies have demonstrated a link between increased circulating ceramides and accumulation (as a major component of ectopic fat) and diabesity, mediated by inflammatory mechanisms (81). These sphingolipids are generated in response to a variety of mediators, including proinflammatory cytokines, oxidative stress, and increased levels of free fatty acids, and would contribute to the state of IR by facilitating inflammatory signaling pathways (i.e., inhibition of insulin action and subsequent glucose uptake through inactivation of Akt pathway and induction of inflammation through activation of the tumor necrosis factor- $\alpha$ axis) $(81,82)$.

Furthermore, it is noteworthy that ceramides differing in the sphingoid base and the fatty acid chain length and saturation are formed in different cell compartments or membranes, by a variety of different mechanisms, at different times, and potentially with distinct biological functions (e.g., dihydroceramides). In turn, the effect of bariatric surgery was most pronounced on specific ceramide species, such as the long-chain C24:0, already associated with the diabese phenotype (83).

In light of recent evidence regarding the role of muscle sphingolipid content in IR (i.e., ceramide enriched with nervonic acid) (56), Mutch et al. (46) speculated that an increase of ceramides in the bloodstream may reflect their mobilization from non-adipose tissues, such as the muscle (26). In confirmation of this hypothesis, elevated intramyocellular lipid deposition was observed in IR and T2D individuals with obesity, while a significant decrease in intramyocellular lipid associated with both reduced circulating sphingolipid molecules and with improved insulin action after RYGB surgery. However, conflicting results have been published (84).

Fatty acid metabolism. Obesity and IR/T2D have been positively associated with decreased fatty acid oxidation, accumulation in the body both in the free and esterified form (lipotoxicity), and to impaired fatty acid elongation and desaturation (higher proportions of saturated species and lower proportions of longer-chain n- 6 and n-3 polyunsaturated fatty acid) (85). In contrast, during dietarybased weight loss a significant reduction of circulating short- and medium-chain SFAs has been described, generally coupled with an improvement in insulin sensitivity $(57,86)$. Blood levels of MUFAs and $\omega-6 / \omega-3$ polyunsaturated fatty acids also decreased significantly in the majority of the studies (Supporting Information Table S3). Interestingly, Perez-Cornago et al. also found that individuals with higher circulating concentrations of palmitoleic acid (C16:1) at baseline experienced a lower reduction in percentage body fat, thus suggesting a certain individual predisposition to responsiveness to the dietary treatment (57). However, any potential causative effect remains controversial (87).

\section{Less-explored metabolic adaptations: Xeno-metabolism and gut microflora}

Finally, metabolic adaptations to both weight loss strategies also included changes in less explored pathways (Supporting Information Table S3), such as in purine/pyrimidine metabolism, xeno, and gut microbial metabolism. Changes in xeno-metabolites including phytochemical derivatives have been observed following both dietary and surgical treatments (e.g., $p$-cresol sulfate) or only postoperatively (i.e., 2-hydroxy(iso)butyric acid) as a consequence of the physical restructuring of the gastrointestinal tract following surgery which involves bypassing much of the proximal small intestine (duodenum and jejunum are the predominant absorption sites for phytochemicals) $(33,34,49)$. Although some of them have been already recognized as early biomarkers of IR and glucose intolerance (88), discrepant results have been collected so far. 
Since the xeno-metabolome is strongly influenced by the intricate relationship between the host metabolism, the diet, and the gut microbiota composition and activity $(24,89)$, starvation, alterations in vitamin status (i.e., biotin, niacin), and changes in the gut microflora following both types of procedures for weight are all possibly implicated. The gut microbiota have been particularly associated with the development of obesity and its comorbidities, mainly due to their contribution in the modulation of several processes including host energy metabolism, gut epithelial permeability, gut peptide hormone secretion, and host inflammatory state. The symbiotic interaction between the gut microbiota and the host is essentially reflected in specific metabolic signatures, and the application of metabolomics has already allowed new insights on the mechanisms linking the gut microbiota composition and activity with disease development (24). Since the microbiota composition may change rapidly in response to both dietary factors and bariatric procedures (49), the effects of a pronounced alteration of gut microbial ecology postoperatively is a particularly hot topic of research currently.

\section{Pitfalls and limitations}

The interpretation of the insights accumulated so far needs to be assessed within the context of the limitations of the reported studies. Some criticisms are relevant to technical limitations of current metabolomic studies in general (e.g., results variability depending on the analytical approaches and platforms employed, the bio-sample selection, preparation, and storage), while others depend on the poorly characterized or highly variable designs of the studies published so far.

From the study design viewpoint, the small scale of the studies and the presence of single-arm designed or parallel-arm studies with poor group-matching criteria were the most common issues hampering biomarker discovery and data exploitation, followed by the common lack of a validation cohort for internal data replication. The limited accessibility of relevant information was another relevant issue (e.g., lack of medication and dietary intake assessment, poor definition of the anthropometric and clinical characteristics of the subjects). Since it is well known that not all subjects with obesity are at the same increased cardiometabolic risk, the metabolic response and effectiveness of a surgical or dietary intervention to treat obesity clearly depend on the metabolic health status of the subjects at baseline (90). Unfortunately, this clinical fact is not reflected in the metabolomic studies carried out to date. Among the reviewed works, in fact, virtually no attempt was observed to stratify individuals with obesity based on their metabolic health phenotype at baseline (i.e., "metabolically healthy" vs. "unhealthy/at risk" phenotypes with obesity), otherwise necessary for personalized followup (91). Also the definition of adiposity was heterogeneous and the assessment of body fat percentage, a recognized predictive marker of cardiometabolic risk and responsiveness to dietary treatments (92), was considered only sporadically. Limited data on the variation of inflammatory mediators following weight loss were also available in the reviewed studies. This prevented the search for associations between the metabolic response to the treatments and the improvement of the proinflammatory state, recognized as a potential mechanism linking adipose tissue expansion and cardiometabolic risk. Similarly, the modest length of the follow-up ( $\leq 1$ year) did not allow us to draw conclusions about the longer-term effects of metabolic surgery, as well as the maintenance phase of any dietary intervention.
Similarly, it is noteworthy that most of the metabolomic studies have focused on examining changes in the overnight-fasted state, although it is trivial that eventual improvement in the metabolic flexibility would manifest more robustly under dynamic challenged conditions, such as an oral glucose tolerance test, a meal or mixed meal tolerance test, or glucose/insulin infusion.

\section{Conclusion}

Moving from association to causation is the next challenge for metabolomics to deepen the link between weight loss treatments and reduction of cardiometabolic risk, and so move biomarker discovery to the next level of clinical effectiveness. To achieve this goal, extrinsic and intrinsic limitations should be faced in the near future, including suboptimal study designs and the prevalent application of targeted approaches (hypothesis-driven and not hypothesisgenerating). $\mathrm{O}$

\section{(C) 2016 The Obesity Society}

\section{References}

1. Newman AB, Lee JS, Visser M, et al. Weight change and the conservation of lean mass in old age: the Health, Aging and Body Composition Study. Am J Clin Nutr 2005;82:872-878.

2. Buchwald H, Estok R, Fahrbach K, et al. Weight and type 2 diabetes after bariatric surgery: systematic review and meta-analysis. Am J Med 2009;122:248-256.

3. Meijer RI, van Wagensveld BA, Siegert CE, Eringa EC, Serné EH, Smulders YM. Bariatric surgery as a novel treatment for type 2 diabetes mellitus: a systematic review. Arch Surg 2011;146:744-750.

4. Kashyap SR, Daud S, Kelly KR, et al. Acute effects of gastric bypass versus gastric restrictive surgery on beta-cell function and insulinotropic hormones in severely obese patients with type 2 diabetes. Int J Obes (Lond) 2010;34:462-471.

5. Adams TD, Gress RE, Smith SC, et al. Long-term mortality after gastric bypass surgery. N Engl J Med 2007;357:753-761.

6. Mingrone G, Panunzi S, De Gaetano A, et al. Bariatric-metabolic surgery versus conventional medical treatment in obese patients with type 2 diabetes: 5 year follow-up of an open-label, single-centre, randomised controlled trial. Lancet 2015; 386:964-973.

7. Masuzaki H, Flier JS. Tissue-specific glucocorticoid reactivating enzyme, 11 betahydroxysteroid dehydrogenase type 1 (11 beta-HSD1)-a promising drug target for the treatment of metabolic syndrome. Curr Drug Targets Immune Endocr Metabol Disord 2003;3:255-262.

8. Shinozaki S, Choi CS, Shimizu N, et al. Liver-specific inducible nitric-oxide synthase expression is sufficient to cause hepatic insulin resistance and mild hyperglycemia in mice. J Biol Chem 2011;286:34959-34975.

9. Bonner C, Farrelly AM, Concannon $\mathrm{CG}$, et al. Bone morphogenetic protein 3 controls insulin gene expression and is down-regulated in INS-1 cells inducibly expressing a hepatocyte nuclear factor 1A-maturity-onset diabetes of the young mutation. J Biol Chem 2011;286:25719-25728.

10. Ashrafian H, Bueter M, Ahmed K, et al. Metabolic surgery: an evolution through bariatric animal models. Obes Rev 2010;11:907-920.

11. Sandoval D. Bariatric surgeries: beyond restriction and malabsorption. Int J Obes (Lond) 2011;35(Suppl 3):S45-S49.

12. Svetkey LP, Stevens VJ, Brantley PJ, et al. Comparison of strategies for sustaining weight loss. JAMA 2008;299:1139-1148.

13. Pournaras DJ, Osborne A, Hawkins SC, et al. Remission of type 2 diabetes after gastric bypass and banding: mechanisms and 2 year outcomes. Ann Surg 2010;252: 966-971.

14. Abbatini F, Rizzello M, Casella G, et al. Long-term effects of laparoscopic sleeve gastrectomy, gastric bypass, and adjustable gastric banding on type 2 diabetes. Surg Endosc 2010;24:1005-1010.

15. Drogan D, Dunn WB, Lin W, et al. Untargeted metabolic profiling identifies altered serum metabolites of type 2 diabetes mellitus in a prospective, nested case-control study. Clin Chem 2015;61:487-497.

16. Munsters MJM, Saris WHM. Body weight regulation and obesity: dietary strategies to improve the metabolic profile. Annu Rev Food Sci Technol 2014;5:39-51.

17. Menni C, Fauman E, Erte I, et al. Biomarkers for type 2 diabetes and impaired fasting glucose using a nontargeted metabolomics approach. Diabetes 2013;62: 4270-4276. 
18. Mihalik SJ, Michaliszyn SF, De Las Heras J, et al. Metabolomic profiling of fatty acid and amino acid metabolism in youth with obesity and type 2 diabetes: evidence for enhanced mitochondrial oxidation. Diabetes Care 2012;35:605-611.

19. Meng Q, Mäkinen V-P, Luk H, Yang X. Systems biology approaches and applications in obesity, diabetes, and cardiovascular diseases. Curr Cardiovasc Risk Rep 2013;7:73-83.

20. Szymańska E, Bouwman J, Strassburg K, et al. Gender-dependent associations of metabolite profiles and body fat distribution in a healthy population with central obesity: towards metabolomics diagnostics. Omics 2012;16:652-667.

21. Suhre K, Meisinger C, Döring A, et al. Metabolic footprint of diabetes: a multiplatform metabolomics study in an epidemiological setting. PLoS One 2010;5: e13953. doi:10.1371/journal.pone.0013953.

22. Roberts LD, Koulman A, Griffin JL. Towards metabolic biomarkers of insulin resistance and type 2 diabetes: progress from the metabolome. Lancet Diabetes Endocrinol 2014;2:65-75.

23. Patti GJ, Yanes O, Siuzdak G. Innovation: metabolomics: the apogee of the omics trilogy. Nat Rev Mol Cell Biol 2012;13:263-269.

24. Palau-Rodriguez M, Tulipani S, Isabel Queipo-Ortuño M, Urpi-Sarda M, Tinahones FJ, Andres-Lacueva C. Metabolomic insights into the intricate gut microbial-host interaction in the development of obesity and type 2 diabetes. Front Microbiol 2015;6:1151. doi:10.3389/fmicb01151.

25. Wang TJ, Larson MG, Vasan RS, et al. Metabolite profiles and the risk of developing diabetes. Nat Med 2011;17:448-453.

26. Floegel A, Stefan N, Yu Z, et al. Identification of serum metabolites associated with risk of type 2 diabetes using a targeted metabolomic approach. Diabetes 2013; 62:639-648

27. Wang-Sattler R, Yu Z, Herder C, et al. Novel biomarkers for pre-diabetes identified by metabolomics. Mol Syst Biol 2012;8:615. doi:10.1038/msb.2012.43.

28. Laferrère B, Reilly D, Arias S, et al. Differential metabolic impact of gastric bypass surgery versus dietary intervention in obese diabetic subjects despite identical weight loss. Sci Transl Med 2011;3:80re2. doi:10.1126/scitranslmed.3002043.

29. Shah SH, Crosslin DR, Haynes CS, et al. Branched-chain amino acid levels are associated with improvement in insulin resistance with weight loss. Diabetologia 2012;55:321-330.

30. Damms-Machado A, Mitra S, Schollenberger AE, et al. Effects of surgical and dietary weight loss therapy for obesity on gut microbiota composition and nutrient absorption. Biomed Res Int 2015;2015:806248. doi:10.1155/2015/806248.

31. Piccolo BD, Comerford KB, Karakas SE, Knotts TA, Fiehn O, Adams SH. Whey protein supplementation does not alter plasma branched-chained amino acid profiles but results in unique metabolomics patterns in obese women enrolled in an 8-week weight loss trial. J Nutr 2015;145:691-700.

32. Campbell C, Grapov D, Fiehn O, Chandler CJ, Burnett DJ, Souza EC, et al. Improved metabolic health alters host metabolism in parallel with changes in systemic xeno-metabolites of gut origin. Claret M, editor. PLoS One 2014;9: e84260. doi:10.1371/journal.pone.0084260.

33. Mutch DM, Fuhrmann JC, Rein D, Wiemer JC, Bouillot J-L, Poitou C, et al. Metabolite profiling identifies candidate markers reflecting the clinical adaptations associated with Roux-en-Y gastric bypass surgery. PLoS One 2009;4:e7905.

34. Gu Y, Zhao A, Huang F, et al. Very low carbohydrate diet significantly alters the serum metabolic profiles in obese subjects. J Proteome Res 2013;12:5801-5811.

35. Piccolo BD, Keim NL, Fiehn O, Adams SH, Van Loan MD, Newman JW. Habitual physical activity and plasma metabolomic patterns distinguish individuals with low vs. high weight loss during controlled energy restriction. J Nutr 2015;145:681-690.

36. Pournaras DJ, Glicksman C, Vincent RP, et al. The role of bile after Roux-en-Y gastric bypass in promoting weight loss and improving glycemic control. Endocrinology 2012;153:3613-3619.

37. Khoo CM, Muehlbauer MJ, Stevens RD, et al. Postprandial metabolite profiles reveal differential nutrient handling after bariatric surgery compared with matched caloric restriction. Ann Surg 2014;259:687-693.

38. Nakatani H, Kasama K, Oshiro T, Watanabe M, Hirose H, Itoh H. Serum bile acid along with plasma incretins and serum high-molecular weight adiponectin levels are increased after bariatric surgery. Metabolism 2009;58:1400-1407.

39. Magkos F, Bradley D, Schweitzer GG, et al. Effect of Roux-en-Y gastric bypass and laparoscopic adjustable gastric banding on branched-chain amino acid metabolism. Diabetes 2013;62:2757-2761.

40. Jüllig M, Yip S, Xu A, Smith G, Middleditch M, Booth M, et al. Lower Fetuin-A, retinol binding protein 4 and several metabolites after gastric bypass compared to sleeve gastrectomy in patients with type 2 diabetes. Singh PK, editor. PLoS One 2014;9:e96489. doi:10.1371/journal.pone.0096489.

41. Aasheim ET, Elshorbagy AK, Diep LM, et al. Effect of bariatric surgery on sulphur amino acids and glutamate. Br J Nutr 2011;106:432-440.

42. Kwon HN, Lee YJ, Kang J-H, et al. Prediction of glycated hemoglobin levels at 3 months after metabolic surgery based on the 7-day plasma metabolic profile. PLoS One 2014;9:e109609. doi:10.1371/journal.pone.0109609.

43. Huang H, Kasumov T, Gatmaitan P, et al. Gastric bypass surgery reduces plasma ceramide subspecies and improves insulin sensitivity in severely obese patients. Obesity (Silver Spring) 2011;19:2235-2240.
44. Heneghan HM, Huang H, Kashyap SR, et al. Reduced cardiovascular risk after bariatric surgery is linked to plasma ceramides, apolipoprotein-B100, and ApoB100/ A1 ratio. Surg Obes Relat Dis 2013;9:100-107.

45. Graessler J, Bornstein TD, Goel D, et al. Lipidomic profiling before and after Roux-en-Y gastric bypass in obese patients with diabetes. Pharmacogenomics $J$ 2013;14:201-207.

46. Ahmad NN, Pfalzer A, Kaplan LM. Roux-en-Y gastric bypass normalizes the blunted postprandial bile acid excursion associated with obesity. Int $J$ Obes (Lond) 2013;37:1553-1559.

47. Simonen M, Dali-Youcef N, Kaminska D, et al. Conjugated bile acids associate with altered rates of glucose and lipid oxidation after Roux-en-Y gastric bypass. Obes Surg 2012;22:1473-1480.

48. Friedrich N, Budde K, Wolf T, et al. Short-term changes of the urine metabolome after bariatric surgery. Omics 2012;16:612-620.

49. Calvani R, Miccheli A, Capuani G, et al. Gut microbiome-derived metabolites characterize a peculiar obese urinary metabotype. Int J Obes (Lond) 2010;34:10951098.

50. Arora T, Velagapudi V, Pournaras DJ, et al. Roux-en-Y gastric bypass surgery induces early plasma metabolomic and lipidomic alterations in humans associated with diabetes remission. PLoS One 2015;10:e0126401. doi:10.1371/ journal.pone.0126401.

51. Lopes TIB, Geloneze B, Pareja JC, Calixto AR, Ferreira MMC, Marsaioli AJ Blood metabolome changes before and after bariatric surgery: a 1(H) NMR-based clinical investigation. Omics 2015;19:318-327.

52. Yu H, Ni Y, Bao Y, et al. Chenodeoxycholic acid as a potential prognostic marker for Roux-en-Y gastric bypass in Chinese obese patients. J Clin Endocrinol Metab 2015; 100:4222-4230.

53. Gralka E, Luchinat C, Tenori L, Ernst B, Thurnheer M, Schultes B. Metabolomic fingerprint of severe obesity is dynamically affected by bariatric surgery in a procedure-dependent manner. Am J Clin Nutr 2015;102:1313-1322.

54. Oberbach $\mathrm{A}$, Blüher $\mathrm{M}$, Wirth $\mathrm{H}$, et al. Combined proteomic and metabolomic profiling of serum reveals association of the complement system with obesity and identifies novel markers of body fat mass changes. J Proteome Res 2011;10:47694788.

55. Dubé JJ, Amati F, Toledo FGS, et al. Effects of weight loss and exercise on insulin resistance, and intramyocellular triacylglycerol, diacylglycerol and ceramide. Diabetologia 2011;54:1147-1156.

56. Kim MJ, Yang HJ, Kim JH, Ahn C-W, Lee JH, Kim KS, et al. Obesity-related metabolomic analysis of human subjects in black soybean peptide intervention study by ultraperformance liquid chromatography and quadrupole-time-of-flight mass spectrometry. $J$ Obes 2013;2013:1-11.

57. Perez-Cornago A, Brennan L, Ibero-Baraibar I, et al. Metabolomics identifies changes in fatty acid and amino acid profiles in serum of overweight older adults following a weight loss intervention. J Physiol Biochem 2014;70:593-602.

58. Tulipani S, Llorach R, Jáuregui O, et al. Metabolomics unveils urinary changes in subjects with metabolic syndrome following 12 -week nut consumption. J Proteome Res 2011;10:5047-5058.

59. Roberts LD, Souza AL, Gerszten RE, Clish CB. Targeted metabolomics. Curr Protoc Mol Biol 2012; Chapter 30:30.2.1-Un24. doi:10.1002/0471142727.mb3002s98.

60. Lynch CJ, Adams SH. Branched-chain amino acids in metabolic signalling and insulin resistance. Nat Rev Endocrinol 2014;10:723-736.

61. McCormack SE, Shaham O, McCarthy MA, et al. Circulating branched-chain amino acid concentrations are associated with obesity and future insulin resistance in children and adolescents. Pediatr Obes 2013;8:52-61.

62. Langenberg C, Savage DB. An amino acid profile to predict diabetes? Nat Med 2011;17:418-420.

63. Tai ES, Tan MLS, Stevens RD, et al. Insulin resistance is associated with a metabolic profile of altered protein metabolism in Chinese and Asian-Indian men. Diabetologia 2010;53:757-767.

64. Newgard CB, An J, Bain JR, et al. A branched-chain amino acid-related metabolic signature that differentiates obese and lean humans and contributes to insulin resistance. Cell Metab 2009;9:311-326.

65. Herman MA, She P, Peroni OD, Lynch CJ, Kahn BB. Adipose tissue branched chain amino acid (BCAA) metabolism modulates circulating BCAA levels. J Biol Chem 2010;285:11348-11356.

66. She P, Van Horn C, Reid T, Hutson SM, Cooney RN, Lynch CJ. Obesity-related elevations in plasma leucine are associated with alterations in enzymes involved in branched-chain amino acid metabolism. Am J Physiol Endocrinol Metab 2007;293: E1552-E1563.

67. Swierczynski J, Sledzinski T, Slominska E, Smolenski R, Sledzinski Z. Serum phenylalanine concentration as a marker of liver function in obese patients before and after bariatric surgery. Obes Surg 2009;19:883-889.

68. Houten SM, Herrema $\mathrm{H}$, Te Brinke $\mathrm{H}$, et al. Impaired amino acid metabolism contributes to fasting-induced hypoglycemia in fatty acid oxidation defects. Hum Mol Genet 2013;22:5249-5261. 
69. Sekhar RV, McKay SV, Patel SG, et al. Glutathione synthesis is diminished in patients with uncontrolled diabetes and restored by dietary supplementation with cysteine and glycine. Diabetes Care 2011;34:162-167.

70. Haeusler RA, Astiarraga B, Camastra S, Accili D, Ferrannini E. Human insulin resistance is associated with increased plasma levels of $12 \alpha$-hydroxylated bile acids. Diabetes 2013;62:4184-4191.

71. Thomas C, Pellicciari R, Pruzanski M, Auwerx J, Schoonjans K. Targeting bile-acid signalling for metabolic diseases. Nat Rev Drug Discov 2008;7:678-693.

72. Staels B, Fonseca VA. Bile acids and metabolic regulation: mechanisms and clinical responses to bile acid sequestration. Diabetes Care 2009;32(Suppl 2):S237$\mathrm{S} 245$.

73. Swann JR, Want EJ, Geier FM, et al. Systemic gut microbial modulation of bile acid metabolism in host tissue compartments. Proc Natl Acad Sci USA 2011; 108(Suppl):4523-4530.

74. Lulsegged A, Saeed E, Langford E, Duffield C, El-Hasani S, Pareek N. Starvation ketoacidosis in a patient with gastric banding. Clin Med 2011;11:473-475.

75. Boden G, Chen X. Effects of fatty acids and ketone bodies on basal insulin secretion in type 2 diabetes. Diabetes 1999;48:577-583.

76. Adimora NJ, Jones DP, Kemp ML. A model of redox kinetics implicates the thiol proteome in cellular hydrogen peroxide responses. Antioxid Redox Signal 2010;13 731-743.

77. Go Y-M, Park H, Koval M, et al. A key role for mitochondria in endothelial signaling by plasma cysteine/cystine redox potential. Free Radic Biol Med 2010;48: 275-283.

78. Jones DP. Redox potential of GSH/GSSG couple: assay and biological significance. Methods Enzymol 2002;348:93-112.

79. Quehenberger O, Armando AM, Brown AH, et al. Lipidomics reveals a remarkable diversity of lipids in human plasma. J Lipid Res 2010;51:3299-3305.

80. Samuel VT, Petersen KF, Shulman GI. Lipid-induced insulin resistance: unravelling the mechanism. Lancet 2010;375:2267-2277.

81. Summers SA. Ceramides in insulin resistance and lipotoxicity. Prog Lipid Res 2006; $45: 42-72$
82. Holland WL, Brozinick JT, Wang L-P, et al. Inhibition of ceramide synthesis ameliorates glucocorticoid-, saturated-fat-, and obesity-induced insulin resistance. Cell Metab 2007;5:167-179.

83. Haus JM, Kashyap SR, Kasumov T, et al. Plasma ceramides are elevated in obese subjects with type 2 diabetes and correlate with the severity of insulin resistance. Diabetes 2009;58:337-343.

84. Skovbro M, Baranowski M, Skov-Jensen C, et al. Human skeletal muscle ceramide content is not a major factor in muscle insulin sensitivity. Diabetologia 2008;51: 1253-1260.

85. Kröger J, Schulze MB. Recent insights into the relation of $\Delta 5$ desaturase and $\Delta 6$ desaturase activity to the development of type 2 diabetes. Curr Opin Lipidol 2012; 23:4-10

86. Schwab U, Seppänen-Laakso T, Yetukuri L, et al. Triacylglycerol fatty acid composition in diet-induced weight loss in subjects with abnormal glucose metabolism - the GENOBIN study. PLoS One 2008;3:e2630. doi:10.1371/journal.pone.0002630.

87. Munro IA, Garg ML. Dietary supplementation with n-3 PUFA does not promote weight loss when combined with a very-low-energy diet. Br J Nutr 2012;108:14661474

88. Gall WE, Beebe K, Lawton KA, et al. alpha-hydroxybutyrate is an early biomarker of insulin resistance and glucose intolerance in a nondiabetic population. PLoS One 2010;5:e10883. doi:10.1371/journal.pone.0010883.

89. Scalbert A, Brennan L, Manach C, et al. The food metabolome: a window over dietary exposure. Am J Clin Nutr 2014;99:1286-1308.

90. Phillips CM, Dillon C, Harrington JM, et al. Defining metabolically healthy obesity: role of dietary and lifestyle factors. Atkin SL, ed. PLoS One 2013;8: e76188. doi:10.1371/journal.pone.0076188.

91. Badoud F, Lam KP, Perreault M, Zulyniak MA, Britz-McKibbin P, Mutch DM. Metabolomics reveals metabolically healthy and unhealthy obese individuals differ in their response to a caloric challenge. PLoS One 2015;10:e0134613. doi:10.1371/ journal.pone. 0134613 .

92. Phillips CM, Tierney AC, Perez-Martinez P, et al. Obesity and body fat classification in the metabolic syndrome: impact on cardiometabolic risk metabotype. Obesity (Silver Spring) 2013;21:E154-E161. 